\title{
Factors Affecting the Purchase of Commercial Health Insurance: Based on Personal Health Status and the Substitution Effect of Social Insurance
}

Wenguang Yu ( $\nabla$ yuwg@sdufe.edu.cn )

Shandong University of Finance and Economics

Qi Wang

Shandong University of Finance and Economics

Guofeng Guan

Shandong University of Finance and Economics

Yujuan Huang

Shandong Jiaotong University

\section{Research Article}

Keywords: commercial health insurance, social basic health insurance, self-rated health

Posted Date: November 11th, 2021

DOI: https://doi.org/10.21203/rs.3.rs-1033408/v1

License: (c) (i) This work is licensed under a Creative Commons Attribution 4.0 International License.

Read Full License 


\title{
Factors Affecting the Purchase of Commercial Health Insurance: Based on Personal Health Status and the Substitution Effect of Social Insurance
}

\author{
Wenguang $\mathrm{Yu}^{1 *}$ Qi Wang ${ }^{2}$ Guofeng Guan ${ }^{2}$ Yujuan Huang ${ }^{3}$ \\ (1. School of Insurance, Shandong University of Finance and Economics, Jinan Shandong \\ 250014, China
}

2. School of Mathematic and Quantitative Economics, Shandong University of Finance and Economics, Jinan Shandong 250014, China

3. School of Science, Shandong Jiaotong University, Jinan Shandong 250357, China)

*Corresponding author: yuwg@sdufe.edu.cn

Background: How health status affects residents' purchase of commercial health insurance and whether social insurance will crowd out the demand of commercial health insurance are important issues related to the development of commercial health insurance. Further, this paper also attempts to answer why people with worse health buy less commercial health insurance through mechanism analysis.

Methods: We used Logit model to analyze the impact of self-rated health level on the purchase of commercial health insurance and the crowding out effect of participating in social health insurance on the purchase of commercial health insurance. In addition, we also successively introduced the respondents with worse and worse health status for regression analysis and analyzed the reasons why the poor health groups reduce the purchase of commercial medical insurance.

Results: When performing regression analysis on whether to purchase commercial health insurance, the regression coefficient of having social health insurance is -0.497 and the regression coefficient of self-rated health level is -0.182 , but the health status has no significant impact on whether to have social health insurance.

Conclusions: The expansion of social basic health insurance has a restraining effect on the promotion of commercial health insurance, and self-rated health level has a negative impact on the purchase of commercial health insurance. The reason why individuals with poor health buy less commercial health insurance is that commercial insurance companies often refuse to cover individuals in poor health.

Key words: commercial health insurance; social basic health insurance; self-rated health JEL: I11, G22

Funding: This research was financially supported by the Key Projcet of National Social Science Foundation of China (No. 21AZD071), the Taishan Scholars Program of Shandong Province (No. tsqn20161041), the Shandong Provincial Social Science Project Planning Research Project(No. 19CQXJ08). 


\title{
Factors Affecting the Purchase of Commercial Health Insurance: Based on Personal Health Status and the Substitution Effect of Social Insurance
}

\begin{abstract}
Background: How health status affects residents' purchase of commercial health insurance and whether social insurance will crowd out the demand of commercial health insurance are important issues related to the development of commercial health insurance. Further, this paper also attempts to answer why people with worse health buy less commercial health insurance through mechanism analysis.
\end{abstract}

Methods: We used Logit model to analyze the impact of self-rated health level on the purchase of commercial health insurance and the crowding out effect of participating in social health insurance on the purchase of commercial health insurance. In addition, we also successively introduced the respondents with worse and worse health status for regression analysis and analyzed the reasons why the poor health groups reduce the purchase of commercial medical insurance.

Results: When performing regression analysis on whether to purchase commercial health insurance, the regression coefficient of having social health insurance is -0.497 and the regression coefficient of self-rated health level is -0.182 , but the health status has no significant impact on whether to have social health insurance.

Conclusions: The expansion of social basic health insurance has a restraining effect on the promotion of commercial health insurance, and self-rated health level has a negative impact on the purchase of commercial health insurance. The reason why individuals with poor health buy less commercial health insurance is that commercial insurance companies often refuse to cover individuals in poor health.

Key words: commercial health insurance; social basic health insurance; self-rated health

JEL: I11, G22

\section{Introduction}

Social basic health insurance and commercial health insurance both play an important role in protecting patients' health expenditures, alleviating residents' financial burden on healthcare, and helping to maintain social stability. However, they are also different in coverage and role. In China's multi-pillar health insurance system, commercial health insurance is an important part and plays a supplementary role to the social basic health insurance. The government encourages enterprises and individuals to solve insurance needs other than social basic health insurance by participating in commercial health insurance and various forms of supplementary insurance. From China's practice, the coverage of social basic health insurance has continued to increase. By the end of 2020, the number of people participating in social basic health insurance has reached 1.36 billion, and the 
coverage has stabilized over $95 \%$ in recent years. In contrast, the development of commercial health insurance is not optimistic, the role played in China's multi-pillar health insurance system is not sufficient. In 2018, the compensation expenditure of commercial health insurance accounts for only $3.01 \%$ of the total health expenditure. By 2020, China's total health expenditure is expected to reach 7230.64 billion yuan, while the compensation expenditure of commercial health insurance is 292.1 billion yuan, the compensation proportion of health insurance only rise to $4.04 \%$. Meanwhile, the total expenditure of social basic health insurance in 2020 is 2,094.926 billion yuan, accounting for $28.97 \%$ of the total national health expenditure, which is a slight decrease from the level of $30.73 \%$ in 2018 , but compared with the compensation of commercial health insurance, it still reflects the large gap between commercial health insurance and social basic health insurance. From the perspective of the development speed of commercial health insurance in recent years, the original premium income of health insurance for the whole year of 2020 is 817.3 billion yuan, which is a year-on-year increase of $15.67 \%$ compared to 706.6 billion yuan in 2019 , the growth rate looks faster. However, from a vertical perspective, the year-on-year growth rates from 2016 to 2019 are respectively $67.72 \%, 8.58 \%, 24.12 \%$, and $29.7 \%$, this growth rate shows a downward trend. Based on the above factors, the role of China's commercial health insurance in meeting people's demands for health care urgently needs to be strengthened. Does the ever-increasing coverage of social basic health insurance make residents more dependent on social basic health insurance and ignore the role of commercial health insurance? What are the individual characteristics that determine the purchase of commercial health insurance? How to improve commercial health insurance so as to enhance the role of China's commercial health insurance in the multi-pillar health insurance system? Answering this series of questions has important academic and practical significance.

The various factors affecting the purchase of commercial health insurance have been discussed enthusiastically by many scholars. For example, Kapur (2020) use the survey data commissioned by the Health Insurance Authority from 2009 to 2017 to examine the determinants of Ireland's demand for private health insurance, the results show that the elderly and sick individuals are more likely to have commercial health insurance. Cantiello et al. (2015) focus on the analysis of the factors affecting the participation of young people aged 18 to 24 in the United States to participate in health insurance, and point out that socioeconomic status, minority status, perceived health, perceived need, and perceived value are important factors that affect whether an individual buys health insurance. In view of the determinants of China's purchase of private health insurance, using the national representative data of the China Household Finance Survey(CHFS) in 2017, Wan et al. (2020) point out predisposing factors such as age, education level, marital status, family size, enabling factors such as family income, SBMI status, geographical factors, family medical expenses medical debts, and needs-based factors are the decisive factors to decide whether to purchase private health insurance. Jin et al. (2016) analyze the purchase of commercial health insurance for groups over 45 in China and its influencing factors, and point out that rural residents are less likely to purchase private commercial health insurance, immigrants, the poor and vulnerable groups are still 
on the edge of the system, and the growing commercial health insurance market does not provide sufficient protection and does not cover the people who need it most.

In addition to personal factors, since both social health insurance and commercial health insurance are insurance products that protect residents' medical expenditures, they have certain common attributes and have certain similarities in their functions. Therefore, there may be competition or cooperation between them. In terms of the relationship between social basic health insurance and commercial health insurance, Marquis and Long (2003) analyze the crowding-out effect of public insurance expansion on private insurance earlier. Their research suggests that the private insurance coverage of low-income people has decreased more than expected in states with expanded public insurance compared with controlled states, indicating some substitution of public coverage for private insurance. In view of this, the government need to take a series of methods to limit the crowd when expanding public insurance. Augurzky and Tauchmann (2011) use the difference-in-differences approach to analyze the impact of policy reforms on the demand for supplementary private insurance. No significant effect on the demand for supplementary health insurance is found. Qin and Liu (2013) take the Obama administration's health care reform as the background and point out that the establishment of the health care safety net in the United States would inhibit individuals from purchasing private insurance. When the community premium rate fails to reflect the value of such resources, not purchasing insurance becomes a rational decision for a sizeable portion of the population. Based on data from the health insurance "Marketplaces" established by the Affordable Care Act, Sen and DeLeire (2018) find that in states where public insurance has expanded, the premiums have decreased $11 \%$, which confirms that private insurance market risk pools are likely to be directly affected by expansions of public insurance. In terms of theoretical analysis, Zhao (2017) studies the possible relationship between social insurance, commercial health insurance and personal welfare through a dynamic general equilibrium analysis and point out that social insurance does not only distort saving and labor supply decisions, but also has a large crowding out effect on the demand for private health insurance. This crowding out effect may be one of the reasons why some Americans are unwilling to buy any commercial health insurance. However, the study also points out that if social insurance is cancelled, the net welfare consequences are still negative in most cases. At the same time, Yuan et al. (2019) also point out that the job tenure has been significantly shortened with the prevalence of the gig economy around the world, and the unemployment-induced social health insurance transition can significantly deteriorate the healthcare utilization of insurance beneficiaries experiencing the transitions from the urban employee basic medical insurance(UEBMI) to the new cooperative medical scheme(NCMS).

In terms of the impact of social insurance expansion on students and adolescents, Gresenz et al. (2012) analyze the effects of states' expansions of Children's Health Insurance Program (CHIP) eligibility to children, the results show that the expansion of CHIP limits the purchase of private health insurance for children from high-income families, and private insurance coverage has been crowded-out. In contrast, Strane et al. (2016) analyze the situation of low-income families, the 
results point out that when the increase of insurance costs exceeded the increase of family income, families relying on employer-sponsored health insurance for their children will turn to provide social insurance for their children. The above literature mainly analyzes the short-term impact of. the expansion of public health insurance plans, Cohodes et al. (2016) analyze the long-term impact of expanding public insurance on children's future education, and confirm that expanding the coverage of commercial health insurance for low-income children has long-term benefits.

For the research on China's social insurance plans and individual commercial health insurance, the earlier study is conducted by Liu et al. (2011), using longitudinal data from the China Health and Nutrition Survey (CHNS, 2000-2006), they find that the New Cooperative Medical Scheme (NCMS) has little impact, but the impact on adults and children is different. When NCMS is launched, the possibility of adults buying private commercial health insurance increased by $2.1 \%$. Moreover, it has a greater positive impact on high-income adult groups. For children, NCMS squeezes out private commercial health insurance for children, especially children from low-income families. In addition to income heterogeneity, health factors also have an important impact. Fang et al. (2019) point out that China's New Cooperative Medical Scheme (NCMS) has a great impact on the medical expenditure of healthy people, but it has no significant impact on people with poor health status. Also using the panel data from the China Health and Nutrition Survey, Hou and Zhang (2017) identify the causal effects of public health insurance expansion on private health insurance development in the case of expansion of the China Urban Residential Basic Medical Insurance (URBMI) program. However, different from the previous research conclusions, the conclusion shows that individuals' purchase of commercial health insurance is not affected by the expansion of URBMI. On the contrary, private commercial health insurance plays an important supplementary role. In order to alleviate the possible crowding-out effect, Zhang et al. (2018) provide an anticrowding solution from two aspects. On the one hand, it is necessary to improve the efficiency and fairness of the medical insurance fund, on the other hand, it is important for insurance companies to innovate insurance products and reduce the insured's overuse of health care.

With the increasing popularity of supplementary health insurance, what is the coverage of private commercial health insurance? Wu et al. (2020) point out that although the coverage of private commercial health insurance has gradually increased, it is unequal in various regions and populations. At the same time, the role of private health insurance in expanding national health insurance in China is limited. In addition, using the 2008-2014 Korea Health Panel, Ko (2020) find that supplementary private insurance can improve the outpatient and inpatient rates, especially for the elderly and low-income people, but the coverage of supplementary private insurance in these groups is very low.

Based on the above literature analysis, this paper puts forward the following research hypotheses.

China's social basic health insurance covers a wide range, which can effectively avoid the problem of adverse selection. As long as you work in a formal unit or live in a community or village 
collectively, you can insure social basic health insurance if there is no special reason, so there may be a crowding-out effect of social basic health insurance. Therefore, Hypothesis 1-1 is proposed.

Hypothesis 1-1: Only purchasing social basic health insurance can meet medical needs, so social basic health insurance has a restraining effect on the purchase of commercial health insurance.

At the same time, it is also possible that the popularization of social basic health insurance has enhanced individual risk awareness and the sense of insurance, thereby promoting residents to purchase more insurance. So, the opposite hypothesis 1-2 is proposed.

Hypothesis 1-2: Social basic health insurance can promote the formation of risk awareness, thus promoting the purchase of commercial health insurance.

As the most important personal characteristic of purchasing commercial health insurance, health status has an important impact on individuals purchasing commercial insurance. Individuals with poor health are more likely to have the need to seek protection, which can promote the purchase of commercial health insurance. Thus, hypothesis 2-1 is put forward.

Hypothesis 2-1: The worse the health level, the stronger the willingness to insure commercial health insurance, so the groups with poor health level buy more commercial health insurance.

However, poor health groups may also be rejected by commercial insurance companies due to physical status, disease history and other factors. Therefore, poor health groups may not be able to participate in commercial health insurance, hence the opposite hypothesis 2-2 is put forward.

Hypothesis 2-2: The group with poor health is not insured by commercial insurance companies due to physical reasons, so people with poor health buy fewer commercial health insurance.

In order to verify the above hypothesis, this paper will select appropriate data and methods for empirical analysis next. The rest of this article is organized as follows. Section 2 is the source of data and the choice of variables. Section 3 describes the methods and results of empirical analysis. Section 4 summarizes the main conclusions of this article and gives relevant suggestions.

\section{Data and Variables}

\subsection{Data Sources}

The data selected for empirical analysis in this paper are from China Health and Retirement Longitudinal study(CHARLS). The survey collected high-quality micro data of middle-aged and elderly families and individuals in China, which is nationally representative. This study is a widely used database in relevant fields, and the data covers the health, health insurance, age, gender, education and other individual characteristics required for this paper. According to the research needs of this paper, relevant data were finally selected from the four parts of demographic backgrounds, health status and functioning, health care and insurance and income in CHARLS in 2018. The above data are matched according to individual ID, and the missing values with incomplete answers are deleted. Finally, a total of 6484 valid data are obtained.

\subsection{Variable Selection}

The dependent variable is whether to buy commercial health insurance. If the individual has purchased commercial health insurance, it is set to 1 , otherwise it is 0 . In terms of the social basic 
health insurance, the respondents who participated in the Urban Employee Basic Medical Insurance, the Urban Residents' Medical Insurance, the New Rural Cooperative Medical Insurance or the Urban and Rural Residents Medical Insurance (the merged Urban Residents' Medical Insurance and New Rural Cooperative Medical Insurance) are set to 1. In terms of health factors, self-rated health is selected as the representative variable of individual health level. For control variables, whether hospitalization occurred in the previous year is selected to measure the utilization of medical services. At the same time, this paper also controls the individual characteristics such as gender, age, urban and rural attributes, educational standards, marital status and so on. The variable names, symbols, and meanings of variables are shown in Table 1.

Table 1. The names, symbols and meanings of variables

\begin{tabular}{|c|c|c|}
\hline Varial & Symbols & The meanings of variables \\
\hline $\begin{array}{l}\text { Whether to purchase } \\
\text { commercial health insurance }\end{array}$ & com_ins & $\begin{array}{l}\text { If commercial health insurance is purchased, it is } 1 \text {; } \\
\text { otherwise, it is } 0\end{array}$ \\
\hline $\begin{array}{l}\text { Whether to purchase social } \\
\text { basic health insurance }\end{array}$ & basic_ins & $\begin{array}{l}\text { If the respondents have participated in at least one } \\
\text { social basic health insurance, it is } 1 \text {, otherwise it is } 0\end{array}$ \\
\hline Self-rated health & health_self & $\begin{array}{l}\text { Self-rated health level, } 1-5 \text { represent very good, } \\
\text { good, generally, poor and very poor respectively }\end{array}$ \\
\hline $\begin{array}{l}\text { Whether hospitalization } \\
\text { occurred in the previous year }\end{array}$ & hos & $\begin{array}{l}\text { If hospitalized in the previous year, it is } 1 \text {, otherwise } \\
\qquad \text { it is set to } 0\end{array}$ \\
\hline Gender & gender & Set to 1 for males and 0 for females \\
\hline Age & age & Numerical variables of respondent's age \\
\hline Urban and rural attributes & location & $\begin{array}{l}\text { Represents the residence of the respondents, and 1-4 } \\
\text { represents cities, urban-rural fringe areas, rural areas } \\
\text { and other regions respectively }\end{array}$ \\
\hline Educational standards & $e d u$ & $\begin{array}{l}\text { Respondents who have received high school } \\
\text { education or above is } 1 \text {, otherwise } 0\end{array}$ \\
\hline Marital status & married & $\begin{array}{l}\text { Respondents who are married are } 1 \text {, and those who } \\
\text { are separated, divorced, and widowed are } 0\end{array}$ \\
\hline
\end{tabular}

\subsection{Descriptive Statistics}

Table 2 shows the descriptive statistics of the whole sample. The average values of com_ins and basic_ins represent the participation rates of commercial health insurance and social basic health insurance respectively. The former is $4.87 \%$ and the latter is as high as $95.79 \%$. The proportion of participating in social basic health insurance is significantly higher than that of commercial health insurance, which reflects that China's social basic health insurance has a wide coverage, while the participation rate of commercial health insurance is low. This result shows that the popularity of commercial insurance in China needs to be strengthened, and it also reflects that the study of the influencing factors of commercial health insurance purchase in this paper has strong 
practical significance for promoting the development of commercial health insurance. In terms of health factors, the average self-rated health is 2.56 , slightly lower than the middle level of indicators 1-5. The proportion of respondents who had been hospitalized in the previous year was $9.86 \%$, indicating that the vast majority of groups were not hospitalized in the past year. In terms of individual characteristics, the distribution of male and female respondents is relatively uniform, men are slightly less than $50 \%$, and the average age of respondents is 61.29 years old, this is because the respondents of CHARLS are mainly middle-aged and elderly groups over 45 years old. The minimum age is 3 , indicating that some respondents are younger than 45 years old. This paper reserves this small number of respondents under the age of 45 . Most of the respondents live in rural areas, and only $14.7 \%$ of them have received high school education or above. Their low education level is more likely to lead to insufficient insurance knowledge and risk awareness, resulting in a low purchase rate of commercial insurance. $88.57 \%$ of the respondents are married. Because this part of the group assumes more family responsibilities, there may be a certain difference from the unmarried group, which affects their willingness to purchase commercial health insurance. Therefore, this paper also controls the marital status of the respondents.

Table 2. Descriptive statistics of the whole sample

\begin{tabular}{ccccc}
\hline Symbols & Mean & Standard deviation & Minimum & Maximum \\
\hline com_ins & 0.0487353 & 0.2153308 & 0 & 1 \\
basic_ins & 0.9578964 & 0.2008411 & 0 & 1 \\
health_self & 2.558452 & 1.004759 & 1 & 5 \\
hos & 0.0985503 & 0.2980802 & 0 & 1 \\
gender & 0.4932141 & 0.49999925 & 0 & 1 \\
age & 61.29411 & 9.773623 & 3 & 98 \\
location & 2.488742 & 0.8324161 & 1 & 4 \\
edu & 0.1469772 & 0.3541105 & 0 & 1 \\
married & 0.8857187 & 0.3181772 & 0 & 1 \\
\hline
\end{tabular}

Table 3 shows the performance of respondents who have not purchased commercial health insurance and those who have purchased commercial health insurance. From the comparison of the mean of the two groups of samples, for those who have not purchased commercial health insurance, the rate of purchasing basic health insurance is $95.9 \%$. while the respondents who have purchased commercial health insurance has a lower rate of basic medical insurance, at $93.7 \%$, indicating that respondents with social basic health insurance buy less commercial health insurance. The average self-rated health of respondents who have not purchased commercial health insurance is 2.57 , while the average self-rated health of respondents who have purchased commercial medical insurance is 2.31. Whether commercial health insurance is purchased or not, the probability of hospitalization in 
the previous year is about $9.8 \%$, which shows that the utilization degree of medical services is basically the same. In terms of individual characteristics, the proportion of male respondents who have not purchased commercial health insurance is about $1 \%$ higher, and the average age is 61.6 years old, while the average age of respondents who have purchased commercial health insurance is only 55.2 years old. From the perspective of urban and rural areas, a larger proportion of respondents who have purchased commercial health insurance live in cities. The respondents who have purchased commercial health insurance also have a higher level of education. Among these respondents, $31.33 \%$ of respondents have received high school education or above, while this proportion is only $13.85 \%$ among respondents who have not purchased commercial health insurance. Finally, respondents who have purchased commercial health insurance are more likely to get married, which may be related to more awareness of family responsibilities.

Table 3. Descriptive statistics of sub samples

\begin{tabular}{cccccc}
\hline \multirow{2}{*}{ Symbols } & \multicolumn{2}{c}{$\begin{array}{c}\text { Respondents who have not purchased } \\
\text { commercial health insurance }\end{array}$} & & \multicolumn{2}{c}{$\begin{array}{c}\text { Respondents who have purchased } \\
\text { commercial health insurance }\end{array}$} \\
\cline { 2 - 3 } \cline { 5 - 6 } & Mean & $\begin{array}{c}\text { Standard } \\
\text { deviation }\end{array}$ & & Mean & $\begin{array}{c}\text { Standard } \\
\text { deviation }\end{array}$ \\
\hline basic_ins & 0.9589818 & 0.1983483 & & 0.9367089 & 0.2438721 \\
health_self & 2.571174 & 1.006284 & & 2.310127 & 0.9421482 \\
hos & 0.0985733 & 0.2981124 & & 0.0981013 & 0.2979233 \\
gender & 0.493677 & 0.5000006 & & 0.4841772 & 0.5005422 \\
age & 61.60814 & 9.800357 & & 55.16456 & 6.779391 \\
location & 2.507782 & 0.8216728 & & 2.117089 & 0.9472897 \\
edu & 0.1384565 & 0.3454065 & & 0.3132911 & 0.4645673 \\
married & 0.8829442 & 0.3215128 & & 0.9398734 & 0.2380983 \\
\hline
\end{tabular}

\section{Empirical Analysis and Results}

\subsection{Basic Regression}

Since the dependent variable of this paper is whether to buy commercial health insurance, this paper selects Logit model for regression analysis. Logit model is suitable for the case where the dependent variable is a binary variable. This paper constructs the following two models to analyze the impact of basic medical insurance and self-rated health level on the purchase of commercial health insurance.

$$
\begin{gathered}
\operatorname{Prob}\left(\text { com_ins }_{i}=1\right)=\alpha_{0}+\alpha_{1} \cdot \text { basic_ins }_{i}+\alpha_{2} \cdot h o s+\sum_{m=3}^{n} \alpha_{m} \cdot X_{i}^{m}+e_{i} \\
\operatorname{Prob}\left(\text { com_ins }_{i}=1\right)=\beta_{0}+\beta_{1} \cdot \text { health_self }_{i}+\beta_{2} \cdot \text { hos }+\sum_{m=3}^{n} \beta_{m} \cdot X_{i}^{m}+u_{i}
\end{gathered}
$$


Where, $X_{i}^{m}$ are a series of control variables representing individual characteristics mentioned above.

Table 4. Regression results of the impact of social basic health insurance and self-rated health on the purchase of commercial health insurance

\begin{tabular}{|c|c|c|c|c|}
\hline & (1) & (2) & (3) & (4) \\
\hline \multirow{2}{*}{ basic_ins } & $-0.457^{*}$ & $-0.497 * *$ & & \\
\hline & $(0.240)$ & $(0.253)$ & & \\
\hline \multirow{2}{*}{ health_self } & & & $-0.258 * * *$ & $-0.182 * * *$ \\
\hline & & & $(0.0574)$ & $(0.0614)$ \\
\hline \multirow{2}{*}{ hos } & & 0.184 & & 0.283 \\
\hline & & $(0.201)$ & & $(0.205)$ \\
\hline \multirow{2}{*}{ gender } & & 0.133 & & 0.106 \\
\hline & & $(0.121)$ & & $(0.121)$ \\
\hline \multirow{2}{*}{ age } & & $-0.0850 * * *$ & & $-0.0841 * * *$ \\
\hline & & $(0.00839)$ & & $(0.00839)$ \\
\hline \multirow{2}{*}{ location } & & $-0.355^{* * *}$ & & $-0.351 * * *$ \\
\hline & & $(0.0690)$ & & $(0.0690)$ \\
\hline \multirow{2}{*}{$e d u$} & & $0.656^{* * *}$ & & $0.617 * * *$ \\
\hline & & $(0.142)$ & & $(0.142)$ \\
\hline \multirow{2}{*}{ married } & & 0.215 & & 0.180 \\
\hline & & $(0.253)$ & & $(0.253)$ \\
\hline \multirow{2}{*}{ Constant } & $-2.538 * * *$ & $2.843 * * *$ & $-2.343 * * *$ & $2.798 * * *$ \\
\hline & $(0.232)$ & $(0.602)$ & $(0.145)$ & $(0.580)$ \\
\hline
\end{tabular}

Note: $* * *, * *$ and $*$ respectively represent significant at the confidence level of $1 \%, 5 \%$ and $10 \%$, and the standard error is in parentheses.

Table 4 shows the impact of basic health insurance and health level on whether to purchase commercial health insurance. The regression results in the first column show that the social basic health insurance can inhibit the purchase of commercial health insurance, that is, after purchasing social basic health insurance, the probability of purchasing commercial health insurance decreases. This result proves that after purchasing social basic health insurance, the demand for commercial insurance brought by the improvement of buyers' risk awareness is not enough to urge buyers to purchase more commercial insurance products. Instead, social insurance crowds out commercial health insurance, which is consistent with the speculation of hypothesis 1-1. The regression results in the third column show that the self-rated health has a significant negative impact on whether to buy commercial health insurance, which verifies the conjecture of hypothesis 2-2. There are two reasons for this situation. On the one hand, people with poor health conditions may have a strong willingness to buy more insurance products., they are eager to buy commercial health insurance products according to adverse selection, but the insurance companies refuse to cover such groups. 
On the other hand, the insurance companies may have launched specific insurance products that patients can insure, and the price of this type of insurance product is higher than that of general products due to the principle of actuarial equivalent. Thus, if the individual's risk awareness does not improve, groups with poor health will not choose such insurance products.

\subsection{Mechanism Analysis}

According to the results of basic regression, is this result due to the refusal of insurance companies or the lack of individual's risk awareness? Since the social basic health insurance does not refuse to insure because of the poor health status of the insured, it can be used to judge whether the health status will affect the individual's willing. If the health status of the respondents does not have a significant impact on the willingness to purchase social basic health insurance, it means that the insurance awareness of different health levels is not significantly different, and it also shows the reason why respondents with poor health buy less commercial health insurance is not from individuals, but from insurance companies. Based on the above analysis, the model (3) is constructed.

$$
\operatorname{Prob}\left(\text { basic_ins }_{i}=1\right)=\beta_{0}+\beta_{1} \cdot \text { health_self }_{i}+\beta_{2} \cdot \text { hos }+\sum_{m=3}^{n} \beta_{m} \cdot X_{i}^{m}+u_{i}
$$

The regression model (3) is similar to the model (2), except that the dependent variable is replaced by the basic health insurance that would not be refused because of poor health. The regression results are shown in Table 5 .

Table 5. Regression results of the impact of self-rated health on the purchase of social basic health insurance

\begin{tabular}{ccc}
\hline & $(1)$ & $(2)$ \\
\hline health_self & -0.0896 & -0.0943 \\
hos & $(0.0619)$ & $(0.0636)$ \\
& & $0.739^{* * *}$ \\
gender & $(0.274)$ \\
& & 0.194 \\
age & & $(0.127)$ \\
location & & 0.0108 \\
& & $(0.00679)$ \\
edu & & -0.114 \\
& & $(0.0837)$ \\
married & & $0.680^{* * *}$ \\
Constant & & $(0.245)$ \\
& $3.358^{* * *}$ & $0.769^{* * *}$ \\
\hline
\end{tabular}


Note: $* * *, * *$ and $*$ respectively represent significant at the confidence level of $1 \%, 5 \%$ and $10 \%$, and the standard error is in parentheses.

The results in Table 5 show that the self-rated health level has no significant impact on the purchase of social basic health insurance. Since the social basic health insurance does not refuse to insure the groups with poor physical conditions, this result shows that individuals with poor health do not have a stronger or more negative willingness to buy health insurance, and the reason why the poor health groups buy less commercial health insurance is mainly due to the refusal of commercial insurance companies. From the perspective of control variables, gender, region and other factors that significantly affect the purchase intention of commercial health insurance do not significantly affect the purchase of social basic health insurance, reflecting that age will not significantly affect the purchase of social basic health insurance. The older age is not restricted to purchase social basic health insurance, but the increase of age significantly reduces the purchase of commercial health insurance, which should be similar to the influence mechanism of health level. In order to further verify it is whether because of the refusal of the insured company that the groups with poor health are less likely to participate in commercial health insurance, this paper also makes a specific analysis according to the self-rated health level. Self-rated health 1 represents very good and 2 represents good. For these two groups, commercial insurance companies generally do not refuse insurance for health reasons. If the above analysis is true, that is to say, the respondents with poor physical condition will not significantly increase or reduce the purchase of commercial insurance, which is only caused by the refusal of insurance companies to cover the unqualified groups, the respondents who self-evaluate their health as very good and good will not significantly affect the purchase of social basic health insurance as their health level changes, and the regression coefficient is not significant. As the group with the worst health is introduced into the regression model, the refusal of insurance companies makes this group unable to buy commercial health insurance, and the coefficient will be significantly negative. Therefore, this paper constructs the following regression model and successively introduces members with worse and worse health status for regression to clarify the reasons why poor health groups reduce the purchase of commercial health insurance.

$$
\operatorname{Prob}\left(\text { com_ins }_{i}=1\right)=\beta_{0}+\beta_{1} \cdot(\text { health_self } \leq G)+\beta_{2} \cdot \text { hos }+\sum_{m=3}^{n} \beta_{m} \cdot X_{i}^{m}+u_{i}
$$

where, $G$ is the critical value of health status with $G=2,3,4,5$ in this paper. When $G=5$, the model is the regression for the whole sample. The regression results are shown in Table 6. In order to clearly show the significance of the regression coefficient, Table 6 lists the specific values of the $\mathrm{Z}$-value and P-value of the regression coefficient.

Table 6. Effects of different samples with different health ranges on whether to purchase commercial medical insurance

\begin{tabular}{lllll}
\hline the scope of samples & coefficient & standard deviation & Z-value & P-value \\
\hline
\end{tabular}




\begin{tabular}{ccccc}
\hline health_self $\leq 2$ & -0.1704 & 0.1748 & -0.97 & 0.330 \\
health_self $\leq 3$ & -0.1169 & 0.0724 & -1.61 & 0.107 \\
health_self $\leq 4$ & $-0.1622^{* *}$ & 0.0641 & -2.53 & 0.011 \\
health_self $\leq 5$ & $-0.1821^{* * *}$ & 0.0613 & -2.97 & 0.003 \\
\hline
\end{tabular}

Note: $* * * * *$ and $*$ respectively represent significant at the confidence level of $1 \%, 5 \%$ and $10 \%$, and the standard error is in parentheses.

It can be seen from the results in Table 6 that when the sample range is only the people with very good and good health, the health level does not significantly affect the purchase of commercial health insurance. At the same time, this conclusion is still valid when considering the groups with very good, good and general self-rated health. This conclusion proves that health status does not significantly affect individuals' purchase of social basic health insurance excluding the reason that commercial insurance companies refuse to cover individuals with poor health. When the group with poor physical condition is introduced into the regression model, this coefficient is significantly negative. With the introduction of groups with very poor physical conditions into the regression model, this negative impact further increases, and the significance increase from $5 \%$ to $1 \%$. The above results prove the previous conclusion once again, that is, health status itself does not significantly affect individuals' willingness to buy commercial health insurance, but because individuals with poor physical condition are rejected by commercial insurance companies, the regression results show that such groups buy less commercial medical insurance.

\subsection{Group-Level Regression Analysis}

The results of sub-sample regression by adjusting the sample range have proved that respondents with poor health buy less commercial health insurance because they are rejected by the insurance company. In order to verify the difference in the impact of different health levels on the purchase of health insurance, this article selects respondents with very good physical health as the baseline group and compares them with other groups. The regression results are shown in Table 7.

Table 7. The regression results of different health levels compared to the baseline group

\begin{tabular}{ccc}
\hline & com_ins & basic_ins \\
\hline Self-rated health & & \\
(Take 1 as the baseline group) & & \\
2 & -0.153 & 0.133 \\
& $(0.173)$ & $(0.213)$ \\
3 & $-0.245^{*}$ & -0.0906 \\
& $(0.146)$ & $(0.167)$ \\
4 & $-0.735^{* * *}$ & -0.301 \\
& $(0.285)$ & $(0.226)$ \\
5 & $-1.377^{*}$ & -0.248 \\
& $(0.728)$ & $(0.418)$ \\
\hline
\end{tabular}




\begin{tabular}{ccc}
\hline hos & 0.314 & $0.756^{* * *}$ \\
& $(0.205)$ & $(0.275)$ \\
gender & 0.110 & 0.195 \\
& $(0.121)$ & $(0.127)$ \\
age & $-0.0837^{* * *}$ & 0.0107 \\
& $(0.00838)$ & $(0.00680)$ \\
location & $-0.351^{* * *}$ & -0.109 \\
edu & $(0.0690)$ & $(0.0839)$ \\
& $0.609^{* * *}$ & $0.662^{* * *}$ \\
married & $(0.143)$ & $(0.246)$ \\
& 0.169 & $0.769^{* * *}$ \\
Constant & $(0.253)$ & $(0.171)$ \\
& $2.560^{* * *}$ & $1.935^{* * *}$ \\
& $(0.574)$ & $(0.535)$ \\
\hline
\end{tabular}

Note: $* * *, * *$ and $*$ respectively represent significant at the confidence level of $1 \%, 5 \%$ and $10 \%$, and the standard error is in parentheses.

The first column of Table 7 shows the differences of other respondents with different self-rated health levels in purchasing commercial health insurance compared with the baseline group ( health_self $=1$ ). Compared to the baseline group with very good self-rated health, the group with good self-rated health has no significant difference in purchasing commercial health insurance. But from the general level of self-rated health level, the coefficients of general, poor and very poor health level are $-0.245,-0.735$ and -1.377 respectively, which are significantly lower than the baseline group. This result confirms the above conclusion that the worse the health level, the less purchase of commercial health insurance. The second column of Table 7 selects the same group as the baseline group, but what is analyzed is the situation of social basic health insurance. According to the regression results in the second column, compared to the group with the very good self-rated health, the performance of individuals with other health levels in purchasing social basic health insurance is not significantly different, which shows that the level of health can't stimulate or inhibit the willingness to buy social basic health insurance.

\subsection{Model Test}

In order to verify the robustness of the model, regression is performed using OLS model, Probit regression model and other methods, and the results and significance are consistent with those reported in this paper. In addition, since health can affect the purchase of commercial insurance, and the purchase of commercial health insurance may also promote health, there may be an endogenous problem of two-way causality. In this paper, blood pressure, blood lipid and blood glucose are selected as instrumental variables for analysis. If the above three indicators are abnormal, it will affect the individual's self-rated health. However, according to the terms of China's commercial health insurance, the insurance company can normally bear the insurance liability for the insured 
whose blood pressure and blood sugar are controlled by drugs. Therefore, the instrumental variables are exogenous. At the same time, the regression results pass the hypothesis of exogenous instrumental variables. By introducing instrumental variables for regression, endogeneity is effectively avoided, and the results still show that the group with worse health has less commercial health insurance, and the P-value is 0.046 , which is significant at the $5 \%$ confidence level.

\section{Conclusions and Recommendations}

Based on the above results, this paper mainly draws the following main conclusions. Firstly, social basic health insurance has a negative impact on the purchase of commercial health insurance, indicating that social basic health insurance can basically meet the medical needs of residents, and the expansion of social basic health insurance has a restraining effect on the promotion of commercial health insurance. Second, self-rated health has a negative impact on the purchase of commercial health insurance. Individuals with poorer health buy less commercial health insurance. Third, from the perspective of influential mechanism, the reason why individuals with poor health buy less commercial health insurance is that commercial insurance companies often refuse to cover individuals in poor health, resulting in that the worse their self-rated health is, the more likely they are to be rejected by insurance companies. Because the social basic health insurance will not refuse the insurance of residents because of their poor health, there is no such situation that the worse their health, the less they buy social basic health insurance.

In view of the above main conclusions, the following suggestions are put forward. On the one hand, insurance companies should pay attention to the development of commercial health insurance products. Commercial insurance products should be different from social insurance in terms of insurance liability, insurance scope and insurance amount, so as to meet the insurance needs other than social insurance. Only in this way can insurance companies promote residents to buy more insurance products other than social security and alleviate the inhibitory effect of the expansion of basic health insurance. On the other hand, commercial insurance companies should meet the insurance needs of poor health groups and provide more insurance products that can be insured by sick groups. According to the standard of physical examination standards, appropriate insurance products are designed for groups with different health levels to ensure that all groups can fairly choose insurance products.

\section{Declarations}

\section{Ethics approval and consent to participate}

Not applicable

\section{Consent for publication}

Not applicable 


\section{Availability of data and materials}

The data set used in this article is from The China Health and Retirement Longitudinal Study (CHARLS), which mainly aims to collect a high quality nationally representative sample of Chinese residents ages 45 and older to serve the needs of scientific research on the elderly. All data will be made public one year after the end of data collection.

\section{Competing interests}

The authors declare that they have no competing interests

\section{Funding}

This research was financially supported by the Key Projcet of National Social Science Foundation of China (No. 21AZD071), the Taishan Scholars Program of Shandong Province (No. tsqn20161041), the Shandong Provincial Social Science Project Planning Research Project(No. 19CQXJ08).

\section{Authors' contributions}

Data curation, Yujuan Huang; Formal analysis, Qi Wang; Methodology, Wenguang Yu; Software, Guofeng Guan; Writing-original draft, Wenguang Yu and Qi Wang. All authors reviewed the manuscript.

\section{Acknowledgements}

Not applicable

\section{References}

1. Kapur K. Private health insurance in Ireland: Trends and determinants. Econ. Soc. Rev. 2020; 51(1): 63-92. https://orcid.org/0000-0002-5240-3800

2. Cantiello J, Fottler MD, Oetjen D, Zhang NJ. The impact of demographic and perceptual variables on a young adult's decision to be covered by private health insurance. Bmc. Health. Serv. Res. 2015; 15(1): 195. https://dx.doi.org/10.1186/s12913-015-0848-6

3. Wan G, Pen Z, Shi Y, Coyte PC. What are the determinants of the decision to purchase private health insurance in China? Int. J. Env. Res. Pub. He. 2020; 17(15): 5348. https://doi.org/10.3390/ijerph17155348

4. Jin Y, Hou Z, Zhang D. Determinants of health insurance coverage among people aged 45 and over in China: Who buys public, private and multiple insurance. Plos One. 2016; 11(8): e0161774. https://dx.doi.org/10.1371/journal.pone.0161774

5. Marquis MS, Long SH. Public insurance expansions and crowd out of private coverage. Med. 
Care. 2003; 41(3): 344-356. https://doi.org/10.1097/01.MLR.0000053017.14257.00

6. Augurzky B, Tauchmann H. Less social health insurance, more private supplementary insurance? Empirical evidence from Germany. J. Policy Model. 2011; 33(3): 470-480. https://dx.doi.org/10.1016/j.jpolmod.2010.12.002

7. Qin X, Liu GG. Does the US health care safety net discourage private insurance coverage? Eur. J. Health Econ. 2013; 14(3): 457-469. https://doi.org/10.1007/s10198-012-0389-4

8. Sen AP, DeLeire T. How does expansion of public health insurance affect risk pools and premiums in the market for private health insurance? Evidence from Medicaid and the Affordable Care Act Marketplaces. Health Econ. 2018; 27(12): 1877-1903. https://doi.org/10.1002/hec.3809

9. Zhao K. Social insurance, private health insurance and individual welfare. J. Econ. Dyn. Control. 2017; 78: 102-117. https://doi.org/10.1016/j.jedc.2017.03.004

10. Yuan B, Li J, Wu L, Wang Z. Multi-Level social health insurance system in the age of frequent employment change: The urban unemployment-induced insurance transition and healthcare $\begin{array}{lllll}\text { utilization in } & \text { China. Healthcare-Basel. 2019; }\end{array}$ https://dx.doi.org/10.3390/healthcare7020077

11. Gresenz CR, Edgington SE, Laugesen M, Escarce JJ. Take-up of public insurance and crowdout of private insurance under recent CHIP expansions to higher income children. Health Serv. Res. 2012; 47: 1999-2011. https://dx.doi.org/10.1111/j.1475-6773.2012.01408.x

12. Strane D, French B, Eder J, Wong CA, Noonan KG, Rubin DM. Low-income working families with employer-sponsored insurance turn to public insurance for their children. Health Affair. 2016; 35(12): 2302-2309. https://doi.org/10.1377/hlthaff.2016.0381

13. Cohodes SR, Grossman DS, Kleiner SA, Lovenheim MF. The effect of child health insurance access on schooling: Evidence from public insurance expansions. J. Hum. Resour. 2016; 51(3): 727-759. https://doi.org/10.3368/jhr.51.3.1014-6688r1

14. Liu H, Gao S, Rizzo JA. The expansion of public health insurance and the demand for private health insurance in rural China. China Econ. Rev. 2011; 22(1): 28-41. https://doi.org/10.1016/j.chieco.2010.08.006

15. Fang CL, He CF, Rozelle S, Shi QH, Sun JY, Yu N. Heterogeneous impacts of basic social health insurance on medical expenditure: Evidence from China's new cooperative medical scheme. Healthcare-Basel. 2019; 7(4): 131. https://doi.org/10.3390/healthcare7040131

16. Hou XH, Zhang J. The effects of public health insurance expansion on private health insurance in urban China. Int. J. Health. Econ. Ma. 2017; 17(3): 359-375. https://doi.org/10.1007/s10754-017-9213-0

17. Zhang Y, Su AN, Liu XX, Zhang Y. Social health insurance vs private health insurance in China: Revisit crowd - out effect based on a multiple mediation analysis. Int. J. Health Plan. M. 2018; 33(4): 996-1012. https://doi.org/10.1002/hpm.2554

18. Wu R, Li N, Ercia A. The effects of private health insurance on universal health coverage 
objectives in China: A systematic literature review. Int. J. Env. Res. Pub. He. 2020; 17(6): 2049. https://doi.org/10.3390/ijerph17062049

19. Ko H. Moral hazard effects of supplemental private health insurance in Korea. Soc. Sci. Med. 2020; 265: 113325. https://doi.org/10.1016/j.socscimed.2020.113325 